\title{
Assessment of three-dimensional set-up errors in pelvic radiation therapy
}

\begin{abstract}
Set-up errors are the inevitable features of the radiation treatment process. Coverage of target volume is a direct function of set-up margins, which should be optimized to prevent inadvertent irradiation of adjacent normal tissues. The aim of this study is to evaluate three dimensional set-up errors and propose optimum margins for target volume coverage in pelvic radiation therapy. 100 Portal images are obtained for the first two fractions for 50 pelvic cancer patients with Hip Fix thermoplastics. Displacements between Digitally Reconstructed Radiographs (DRR) and Electronic Portal Images (EPI) are estimated and analyzed along major three axes. Mean displacements, population systematic and random errors and three dimensional vectors of displacements are calculated. Set-up margins are calculated using published margin recipes; International Commission on Radiation Units and measurements (ICRU), Stroom and van Herk. The random errors are 0.2339, 0.1603, and $0.1628 \mathrm{~cm}$ respectively along antero-posterior (AP), supero-inferior (SI) and mediolateral (ML) axes. Similarly; the systematic errors are $0.2698,0.3284$, and $0.2568 \mathrm{~cm}$ along AP, SI and ML axes respectively. The mean displacement in vertical, longitudinal and lateral axes are $0.103,-0.177,0.075 \mathrm{~cm}$ respectively and standard deviation in vertical, longitudinal and lateral axes are $0.2698,0.3284,0.2568$ respectively for pelvic cancer. Based on ICRU report 62 , the clinical target volume to planning target volume margins are $0.357,0.365$ and $0.304 \mathrm{~cm}$ along AP, SI and ML axes respectively. The corresponding values are 0.703 , 0.769 and $0.627 \mathrm{~cm}$ with Stroom's formula and $0.838,0.933$ and $0.756 \mathrm{~cm}$ with van Herk's formula. The results suggest that there is a significant difference within the three major axes. Approximately $22 \%$ of portal images have exceeded the tolerance limit $(>5 \mathrm{~mm})$ of pelvic cancer patients with Hip Fix thermoplastics. Calculated set-up margins are compared well with published margin recipes.
\end{abstract}

Keywords: set-up errors, random error, systematic error, digitally reconstructed radiographs, electronic portal images, hip fix thermoplastics
Volume I Issue 2 - 2014

\author{
LThasanthan, ' WRO Piyasena,' AMC Croos,' \\ R Dhanushia, ${ }^{2}$ PP Narayanan ${ }^{3}$ \\ 'Department of Radiography/ Radiotherapy Faculty of Allied \\ Health Sciences, University of Peradeniya, Sri Lanka \\ 2Ballarat Regional Integrated Cancer Center, Australia \\ ${ }^{3}$ Olivia Newton-John Cancer and Wellness Center, Australia
}

Correspondence: $L$ Thasanthan, Department of Radiography/ Radiotherapy Faculty of Allied Health Sciences, University of Peradeniya, Sri Lanka, Tel 8I399962I, Fax 8I 2387394, Email thasanth@gmail.com

Received: August 10,2014 | Published: September 30, 2014
Abbreviations: DRR, digitally reconstructed radiographs; EPI, electronic portal images; ICRU, international commission on radiation units and measurements; AP, antero-posterior; SI, superoinferior; ML, medio-lateral

\section{Introduction}

The goal of Radiation Therapy for cancer is to reliably provide optimal target coverage and dose level to the gross tumor volume (GTV) and planning target volume (PTV) while minimizing the toxicity to organs at risk (OAR). Improved radiotherapy planning and treatment methods such as three dimensional conformal radio therapies (3DCRT) are achieving this goal by $3 \mathrm{D}$ anatomic information and target volume coverage. The concept of conformal dose distribution has also been extended to include clinical objectives such as maximizing tumor control probability (TCP) and minimizing normal tissue complication probability (NTCP). ${ }^{1}$ The coverage of target volume is a direct function of set-up margins, which should be optimized to prevent inadvertent irradiation of adjacent normal tissues. PTV that encompasses the clinical target volume (CTV) with some margins to account for such uncertainties in patient positioning, organ motion and beam geometry.

Set-up errors are of two categories; random errors and systematic errors. Random errors blur the dose distribution, whereas systematic errors cause a shift of the cumulative dose distribution relative to the target. The random errors represent day-to-day variation in the setup of patient, occur only once and as the name implies is unpredictable. ${ }^{4}$
The systematic errors are more dangerous since they are in affect during all radiation delivery sessions and may inflict serious damage destroying either healthy tissues or provoking tumor recurrence. Since the systematic errors move all measurements in one direction, it may be easily evaluated. ${ }^{5}$ The evaluation of set-up errors is based on the comparison between a portal image and a reference image, which may be a digitized simulation film, a Digital Reconstructed Radiograph (DRR), or another portal image. In this study comparisons are done using portal image and DRR. The aim of this study is to evaluate three dimensional set-up errors and propose optimum margins for target volume coverage in pelvic radiation therapy.

\section{Materials and methods}

In this study 50 pelvic cancer patients with Hip Fix thermoplastics device are included through randomized cluster sampling. Adequate immobilization and positioning devices (pelvic board, head rest and foot rest) are applied to selected patients and pre-treatment portal images are obtained for the first two fractions per patient using Portal Vision as 1000 which was amorphous silicon based EPID system consisting of a detector screen and optical chain. It was mounted isocentrically on the Linear Accelerator with a detector size of $30 \times 40 \mathrm{~cm}$. 100 portal images are acquired at orthogonal gantry angles $0^{\circ}$ and $90^{\circ}$ using a typical exposure time $1 \mathrm{MU}$ at a dose rate of $300 \mathrm{MU} / \mathrm{min}$ and 6MV energy.

DRRs are used as a reference image for comparison with portal images which are reconstructed from computed tomography (CT) 
images. Evaluation of set-up errors is done by defining reproducible and easily identifiable bony landmarks of the treatment field; each in anterior and lateral images. For the purpose of documentation and analysis, anterior, superior, and right sided shifts are coded as positive shifts and posterior, inferior and left-sided shifts as negative shifts. Displacements between DRRs and electronic portal imaging device (EPID) images are estimated and analyzed along three major axes by matching rigid bony structures. Rotational errors are not assessed in the study. The dose delivered by portal imaging is not taken into consideration in calculating the final total dose received by any patient.

Mean displacements, population systematic $(\Sigma)$ and random errors $(\sigma)$ and set-up margins are calculated using published margin recipes; International Commission on Radiation Units and measurements (ICRU) report $62\left(\operatorname{sqrt} \Sigma^{2}+\sigma^{2}\right),{ }^{3}$ Stroom $(2 \Sigma+0.7 \sigma)^{6,7}$ and van Herk $(2.5 \Sigma+0.7 \sigma)^{8,9}$

\section{Results and Discussion}

The values of displacements of pelvic cases are measured and assessed in vertical (Antero-posterior), longitudinal (Supero-inferior) and lateral (Medio-lateral) directions. The MINITAB statistical package and Microsoft office Excel software are used to analyze data. The mean displacements, systematic and random errors in pelvic cancer patients, along major three axes are given in (Table 1).

Table I Overall mean and set-up errors for pelvic cancer along three major axes

\begin{tabular}{llll}
\hline & \multicolumn{3}{l}{ 3-D Translations $(\mathbf{c m})$} \\
\cline { 2 - 4 } & Vertical & Longitudinal & Lateral \\
\hline Overall Mean & 0.103 & -0.177 & 0.075 \\
Random Error & 0.2339 & 0.1603 & 0.1628 \\
Systematic Error & 0.2698 & 0.3284 & 0.2568 \\
\hline
\end{tabular}

The overall mean values of displacement are $0.1030,-0.1770$ and $0.0750 \mathrm{~cm}$ along vertical, longitudinal and lateral axes respectively. The mean displacements for all images in the major three axes were compared using Kruskal-Wallis test analysis with the threshold $\alpha=0.05$ (which corresponds $95 \%$ confidence). Comparisons showed significant differences within the major three axes. Among all patients $78 \%$ of portal images are within the boundary (Pelvis $<5 \mathrm{~mm}$ ) and portal images outside the boundary are accepted after fine tuning.

Table 2 CTV-PTV margins for pelvic cancer

\begin{tabular}{llll}
\hline Direction & ICRU 62 & Stroom & van Herk \\
\hline Vertical & 0.357 & 0.703 & 0.838 \\
Longitudinal & 0.365 & 0.769 & 0.933 \\
Lateral & 0.304 & 0.627 & 0.756 \\
\hline
\end{tabular}

Scatter plot of displacements for all observations in all three directions are given in (Figure 1-3). Calculated CTV to PTV margins according to ICRU Report 62, Stroom and van Herk's formulae are given in (Table 2). Significant displacements are observed for the pelvic cancer patients. Therefore, this group of patients require a special care and should be monitored more often using EPID. The good set-up accuracy can be achieved for pelvic cancer patients using thermoplastic immobilization device with ankle rest or foot rest by preventing pelvic and leg rotation within the immobilization device.

Several mathematical formulae have been recommended for generating CTV-PTV margins. The ICRU 62 assumes that random and systematic errors have an equal effect on dose distribution, which may not necessarily be the case. Using coverage probability matrices and dose-population histograms, Stroom et al., ${ }^{7}$ van Herk et al., ${ }^{9}$ have suggested formulae which incorporate this differential effect. EPID is a useful tool for a fast and reliable assessment and correction of various geometrical errors during the whole process of radiotherapy. It is advisable to apply EPID in radiation therapy departments where the 3-DCRT is introduced.

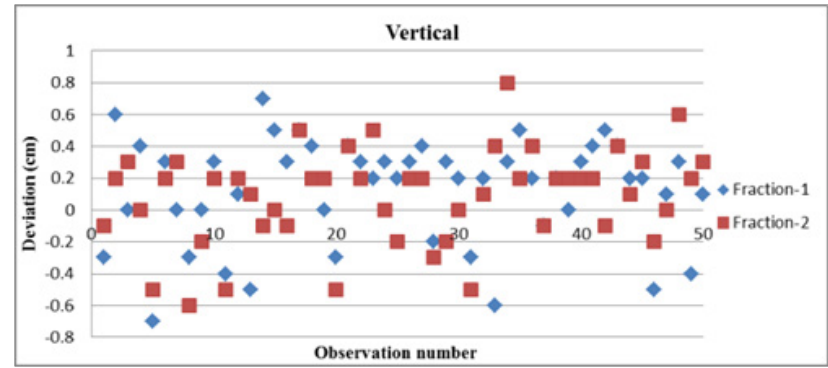

Figure I Scatter plot of displacements in vertical direction.

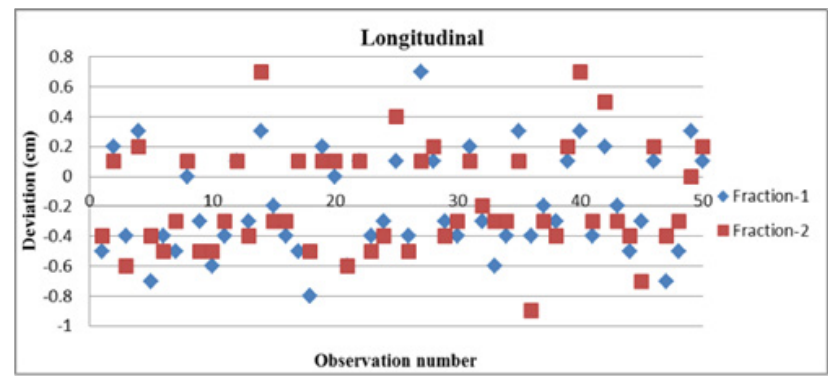

Figure 2 Scatter plot of displacements in longitudinal direction.

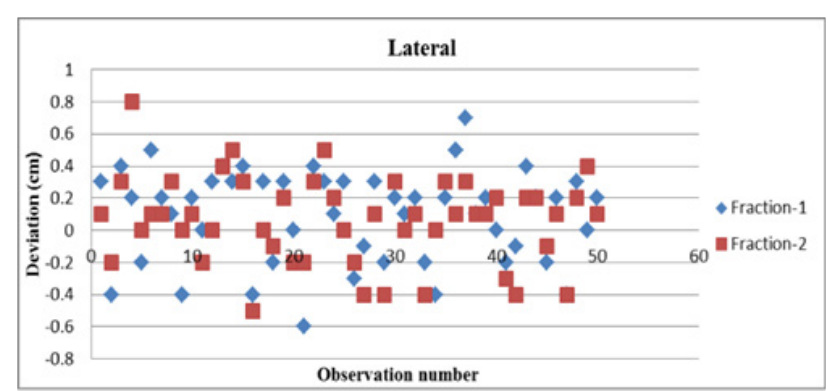

Figure 3 Scatter plot of displacements in lateral direction.

If pertinent, stable structures in relation to three major axes and inserting fiducially markers would be useful to assess field position. Offline correction is very effective in managing the systematic component of set-up errors but has little effect on the random component and complete removal of both systematic and random errors can be achieved by on-line position verification since it is a logical and feasible extension of all EPI protocols giving significant advantages that far outweigh the disadvantages. ${ }^{10-12}$ Modern imageguided radiation therapy (IGRT) systems track the changes of target positioning at the time of the treatment and deliver the accurate and adequate dose to the target. IGRT is a novel accurate technique for conform the dose distribution within the tumor and rapid dose gradients and also competes well to manage three-dimensional set-up errors.

\section{Conclusion}

It is observed that, there is a significant difference in displacements for major three axes. Approximately $78 \%$ of displacements are within the tolerance uncertainties. Among calculated CTV PTV margins 
according to three formulae (Table 2), the margins which are calculated according to ICRU Report 62 is suggested as a optimum margins for target volume coverage which is $<5 \mathrm{~mm}$ in all three directions. From this study, to manage random and systematic errors effectively it is suggested to obtain pre-treatment portal images weekly.

\section{Acknowledgments}

None.

\section{Conflicts of interest}

Authors declare there are no conflicts of interest.

\section{References}

1. Faiz MK. The physics of radiation therapy. 3rd ed. Lippincott Williams \& Wilkins: Philadelphia, USA; 2003. 467 p.

2. International Commission on Radiation Units and Measurements. Prescribing, recording and reporting photon beam therapy. In ICRU Report, 50 Bethesda, MD: ICRU Publications; 1994.

3. International Commission on Radiation Units and Measurements. Prescribing, recording and reporting photon beam therapy (Supplement to ICRU report 50). In ICRU Report, 62 Bethesda, MD: ICRU Publications; 2000.

4. Gupta T, Chopra S, Kadam A, et al. Assessment of three-dimensional setup errors in conventional head and neck radiotherapy using electronic portal imaging device. Radiat Oncol. 2007;14:2-44.
5. van Herk M, Barrilot I, Bel A, et al. Geometrical uncertainties in conformal radiotherapy-and how to deal with them. 6th International Workshop on Electronic Portal Imaging: Brussels; 2000.

6. Stroom JC, Heijmen BJ. Geometrical uncertainties, radiotherapy planning margins, and the ICRU-62 report. Radiother Oncol. 2002;64(1):75-83

7. Stroom JC, de Boer HC, Huizenga $\mathrm{H}$, et al. Inclusion of geometrical uncertainties in radiotherapy treatment planning by means of coverage probability. Int J Radiat Oncol Biol Phys. 1999;43(4):905-919.

8. Van Herk M. Errors and margins in radiotherapy. Semin Radiat Oncol. 2004;14(1):52-64.

9. Van Herk M, Remeijer P, Rasch C, et al. The probability of correct target dosage: dose-population histograms for deriving treatment margins in radiotherapy. Int J Radiat Oncol Biol Phys. 2000;47(4):1121-1135.

10. Alasti H, Petric MP, Catton CN, et al. Portal imaging for evaluation of daily on-line setup errors and off-line organ motion during conformal irradiation of carcinoma of the prostate. Int J Radiat Oncol Biol Phys. 2001;49(3):869-884.

11. Stryker JA, Shafer J, Beatty RE. Assessment of accuracy of daily setups in prostate radiotherapy using electronic imaging. Br J Radiol. 1999;72(858):579-583.

12. Mark M, Aldo R, Steve M, et al. Online versus offline corrections: opposition or evolution? A comparison of two electronic portal imaging approaches for locally advanced prostate cancer. The Radiographer. 2006;53(1):24-28 